\title{
FORCED VIBRATIONS ANALYSIS OF A CANTILEVER BEAM USING THE VISION METHOD
}

\author{
Mateusz Romaszko, Bogdan Sapiński, Andrzej Sioma \\ AGH University of Science and Technology, Faculty of Mechanical Engineering and Robotics, Kraków, Poland \\ e-mail:matek@agh.edu.pl; deep@agh.edu.pl; andrzej.sioma@agh.edu.pl
}

\begin{abstract}
This article presents an analysis of forced vibrations of a homogeneous cantilever beam using a vision method. Based on measurements, displacements of defined beam points have been determined as a function of time in directions perpendicular to and along the beam axis together with the trajectory of these points in the plane of the beam transverse vibrations. A model describing the displacement of the beam with a moving holder (kinematic excitation) is presented along with formulas defining motion of points in the plane of the beam transverse vibrations, which have been verified experimentally by recording beam vibrations using a specialised vision system.
\end{abstract}

Keywords: cantilever beam, vision system, vibration, mode shapes

\section{Introduction}

Contactless methods are currently dominant in the measurement of vibrations of continuous systems. They perform particularly well in the analysis of vibrations of so-called lightweight constructions, in which the application of conventional measuring systems utilising e.g. accelerometers is not viable. Accelerometers introduce additional mass, which causes changes in both damping and rigidity of the studied system. The solution is to use contactless optical sensors (Siram et al., 1992; Freymann et al., 1996; Chen et al., 2003; Van der Auwerwer et al., 2002; Moreno et al., 2005) as well as magnetic and ultrasound sensors. The broad and increasing application of visual methods (Ma et al., 2004; Trucco and Verri, 1998) for analysis of vibrations of continuous systems can be observed. An example might be the determination of free vibration forms and experimental modal analysis (Kohut and Kurowski, 2005; Peeters et al., 2004; Schmidt et al., 2003).

Studies of free and forced vibrations of beams have already been conducted in form of three-layered single- and multi-segments beams filled with magnetorheological fluid (Snamina et al., 2012a; Romaszko et al., 2011). In previous studies, either two laser sensors or one laser vibrometer have been used to measure displacements (Sapiński et al., 2010), with transverse vibrations of beams being measured at only two points.

In this study, in order to increase the number of measuring points describing the beam vibrations, it has been decided to perform the measurement using a vision system. This enabled contactless recording of vibrations of the entire beam and synchronisation of the measurement of all characteristic points determined on the basis of theoretical analysis.

The tested object is a homogeneous cantilever beam made of aluminium. The forced vibrations of the beam with a moving holder are analysed using a vision system specially configured for the purposes of this study which enabled recording the displacement of defined beam points. Three forms of the beam eigenfunctions (modes) are selected for the analysis. Based on recorded data, the dislocations of defined points are determined in the plane of the beam vibrations. A model of the cantilever beam with kinematic excitation is presented and modes for 
the appropriate boundary conditions are determined. The results obtained from experiments are compared with the results obtained from the model.

\section{The vision method}

The vision method of measurement of beam vibrations is based on the recording of a sequence of images followed by analysis of each image using an algorithm described in the next part of the paper. The purpose of this analysis is to determine vibration parameters describing the studied beam. The amplitude of vibrations is determined for each of the characteristic points of the beam in two directions: perpendicular to and along the beam axis. Measurements using the vision method are carried out in three stages:

- analysis of the task and selection of hardware configuration of the vision system,

- recording of the sequence of images at the laboratory test stand,

- development of an image analysis algorithm, performance of measurements and archiving of measurement data.

The first stage encompasses analysis of the measuring task and selection of hardware configuration of the vision system. The optical system is selected, taking into account the dimension of the beam fastened to the shaker. The image is set up so that the entire cantilever beam and moving element of the shaker are visible. The parameters of the vision system are chosen to enable image recording with a sampling rate of 1000 frames/second and an exposure time equal to $400 \mu$ s for a single frame. Images are recorded with the use of a vision system equipped with a HSC (high speed camera) matrix with a resolution of $1200 \times 1000$ pixels. The sampling rate is selected with regard to the resolution of the matrix, the size of the studied object and the lighting conditions at the experimental set-up. These parameters are selected on the basis of tests; their selection is dictated by the necessity of obtaining appropriate contrast between three elements of the filmed scene: the beam, markers, and background. Figure 1 presents a recorded view in which the beam and applied markers, as well as the background, enabling observation of both the beam and the markers, are visible. To carry out the studies, the markers are applied to the beam at seven characteristic points.

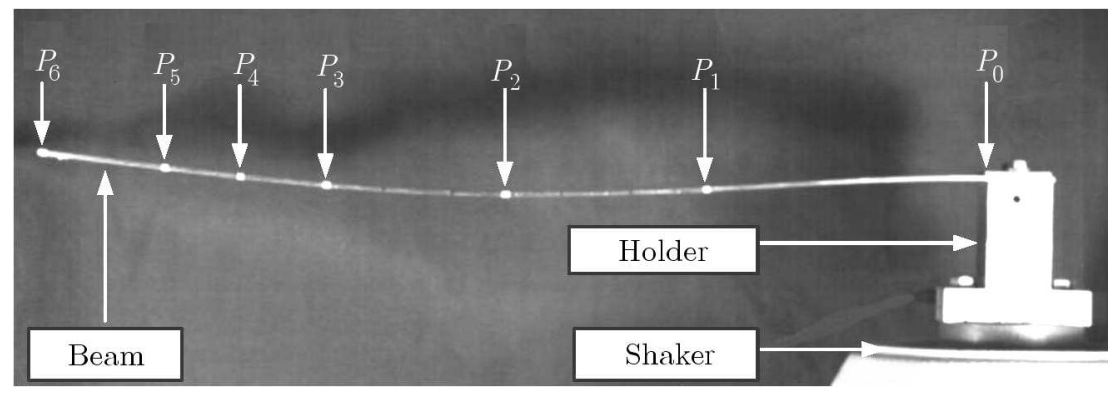

Fig. 1. Measurement points in the beam

Due to small dimensions of the beam side surface, miniature markers, visible in form of white spots, are prepared and applied to this surface (Fig. 1). The placement of markers describing measuring points is selected to enable observation of the free displacement of the end of the beam, $P_{6}$, and the displacement of the beam moving holder (shaker), $P_{0}$. In addition, the positions at which nodes and anti-nodes are located have been marked for the second and third mode. These locations are determined based on formulas defining the eigenfunctions, derived earlier. In this way, the successive points are selected: $P_{1}-P_{5}$. The vision system is installed at 
the experimental set-up and calibrated (Gawlik et al., 2004; Kowal and Sioma, 2009; Sioma. 2013).

The second stage of measurements using the vision method involves the recording of a sequence of beam vibration images at the experimental set-up. The homogeneous cantilever beam is made of aluminium, with a length of $l=400 \mathrm{~mm}$, width of $b=30 \mathrm{~mm}$, and a thickness of $h=2 \mathrm{~mm}$. A diagram of the experimental set-up is presented in Fig. 2. One end of the beam is fastened to the holder of the moving part of the TIRA S514 electrodynamic shaker. The core of the shaker moves in the manner described by the variable $u(t)$ in the direction perpendicular to the beam axis. In this way, an excitation is effected. The shaker is controlled using the LMS SCADAS III module supported by a computer with LMS TestLab software. The M345C03 piezoelectric accelerometer from PCB Piezoelectronics company fastened to the shaker holder is used to control the shaker. The control signal is amplified by the TIRA BAA500 amplifier (Sapiński et al., 2011; Snamina et al., 2012b). The experiments have been conducted for a sinusoidal excitation with a constant frequency $f: 9.15,61.12$ and $165.71 \mathrm{~Hz}$. The amplitudes of excitation have been assumed to be: $0.85,0.81,0.11 \mathrm{~mm}$. The excitation frequency has been selected so as to be similar, respectively, to the first, second, and third resonance frequency of the beam. Beam vibrations have been registered for the first three modes.

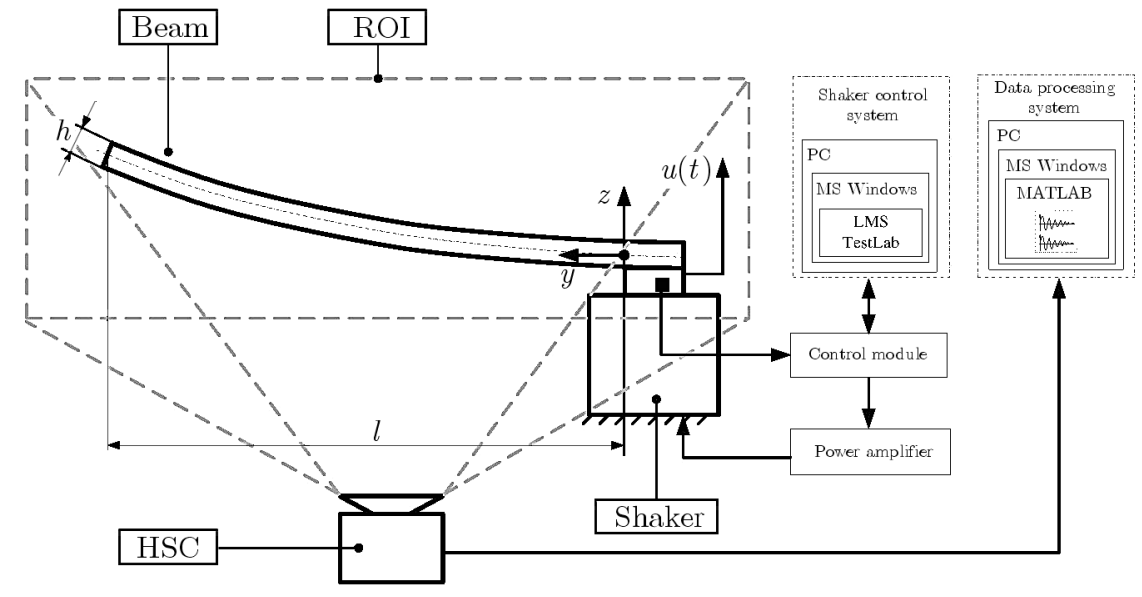

Fig. 2. Schematic diagram of the experimental set-up

The third stage of measurements of the beam vibrations using the vision method involves the development of an algorithm for analysis of the recorded sequence of images. The purpose of the analysis is to determine the displacements of each of the points visible on the image defining the amplitude of vibrations observed for each of the three frequencies. The recorded images are subjected to preliminary processing, and the image intensity is scaled in order to improve the visibility of the marker applied to the beam. Elimination of noise and accidental flaws in form of reflexes observed on the image is done during preliminary processing through the execution of context transformations. The image-scaling procedure is performed after preliminary processing in order to convert point positions registered in pixels to values expressed in millimeters. For the thus prepared image, 7 ROIs (regions of interest) are defined individually for each point. An example of an image presenting the definition of ROIs for the two terminal points of the beam is presented in Fig. 3. The definition of ROIs made it possible to adjust the parameters of COG (center of gravity) determination for the marker of every point to the conditions present in its surroundings. Next, parameters of the segmentation process are selected for every ROI, which enables determination of the COG for each marker applied to the beam so as to locate it in the neutral layer of the beam. In the next step of image analysis, an auxiliary coordinate system is defined. Point $P_{0}$ is placed simultaneously at the position of the beam holder and the axis of symmetry. Its position is determined based on analysis of the edge of the beam 
fastening system on the shaker. This point serves to define the centre of the $y z$ coordinate system (Figs. 2 and 3). The $y$ axis of the coordinate system is positioned horizontally, the $z$ axis vertically. In this way, the coordinates of point $P_{0}(0,0)$ are obtained. Due to the necessity of introducing the marker of point $P_{6}$ onto the edge of the beam and considering the algorithm for determination of the coordinates of the centre of the marker of this point, point $P_{6}$ is placed at a distance of $\Delta=3.6 \mathrm{~mm}$ from the end of the beam (Fig. 3). Using the coordinate system and the determined positions of markers for points $P_{0}-P_{6}$, the coordinates of points are determined for the zero position of the beam: $P_{0}(0,0), P_{1}(117,0), P_{2}(201,0), P_{3}(276,0), P_{4}(312,0), P_{5}(344,0)$, $P_{6}(396.4,0)$. It is assumed that the beam zero position would occur when its longitudinal axis coincides with the $y$ axis. Based on analysis of point positions on successive recorded images, the trajectory of their motion in the plane of the beam transverse vibrations $y z$ is determined. The final step of the third stage of measurements using the vision method is the processing and saving of the results of measurements. The center-of-gravity position parameters determined on successive images and described in the defined $y z$ coordinate system are saved as a csv file. The use of this type of file made it possible to import the data into MATLAB software, in which analysis of the data obtained from the vision method is continued (Fig. 2).

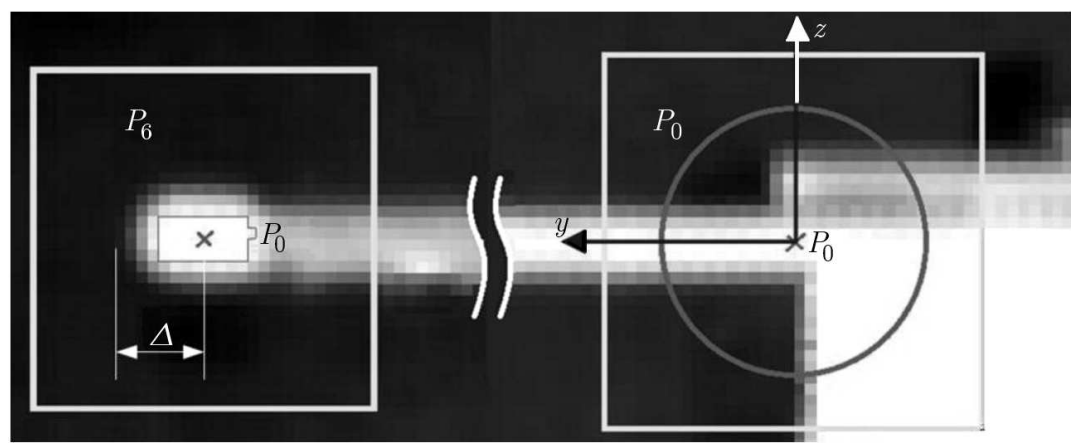

Fig. 3. Beam with the assumed coordinate system and points $P_{0}$, and $P_{6}$

To sum up, the third stage of measurements can be presented in form of an algorithm, a diagram of which is shown in Fig. 4. The main steps of the algorithm can be distinguished on this diagram: preliminary image processing and images preparation for measurements, performance of measurement on an image, archiving of measurement data.

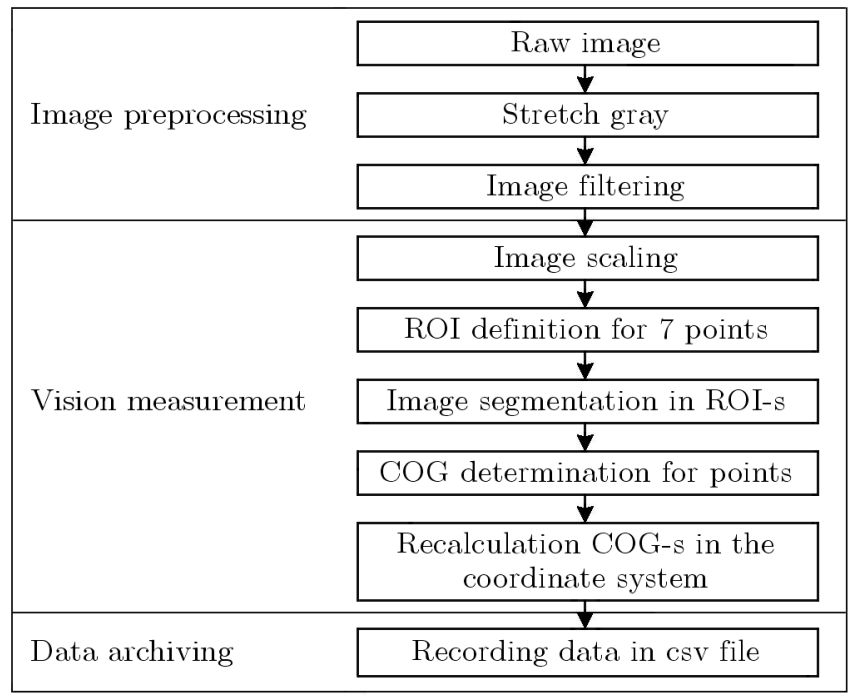

Fig. 4. Algorithm of the vision method 


\section{Theoretical analysis}

The transverse vibrations of an aluminium cantilever beam are considered. One of the beam theories assumes that straight and cross-sections relative to the beam axis remain flat and perpendicular even after the beam is deformed. As a result of bending, cross-sections are subject to rotation, and the fibres of one layer of the beam are subject to tension while those of the second layer are compressed. However, there is a layer that does not sustain any deformation: the neutral layer. The presented assumptions are known as the Euler-Bernoulli hypothesis (Meirovitch, 1997, 2001). The equation based on these assumptions describing transverse vibrations $w=w(y, t)$ of a beam is given by the formula

$$
\frac{\partial^{2} w}{\partial t^{2}}+c^{2} \frac{\partial^{4} w}{\partial x^{4}}=0 \quad c=\sqrt{\frac{E I}{\rho A}}
$$

where $E$ is Young's modulus, $I$ - moment of inertia, $\rho$ - material density, $A$ - area of cross-section. Using the method of separation of variables, the solution of the transverse beam vibration equation is written as

$$
w(y, t)=W(y) T(t)
$$

where $W(y)$ is the eigenfunction and $T(t)$ function describing beam displacement as a function of time $t$. After inserting expression (3.2) into (3.1) 1 and after transformation, the eigenvalue equation is obtained

$$
W^{I V}-\beta^{4} W=0 \quad \beta^{4}=\frac{\omega^{2}}{c^{2}}=\omega^{2} \frac{\rho A}{E I}
$$

The $\left({ }^{I V}\right)$ symbol designates the derivative with respect to the $y$ coordinate. The solution to the eigenvalue equation is the following function

$$
\begin{aligned}
& W(y)=C_{1}(\cos \beta y+\cosh \beta y)+C_{2}(\cos \beta y-\cosh \beta y)+C_{3}(\sin \beta y+\sinh \beta y) \\
& \quad+C_{4}(\sin \beta y-\sinh \beta y)
\end{aligned}
$$

The constants $C_{1}, \ldots, C_{4}$ are determined after the appropriate boundary conditions have been accounted for. For the cantilever beam under consideration, they are as follows

$$
\begin{array}{ll}
\left.w(y, t)\right|_{y=0}=0 & \left.\frac{\partial w(y, t)}{\partial y}\right|_{y=0}=0 \\
-\left.E I \frac{\partial^{2} w(y, t)}{\partial y^{2}}\right|_{y=l}=0 & -\left.E I \frac{\partial^{3} w(y, t)}{\partial y^{3}}\right|_{y=l}=0
\end{array}
$$

After determination of the constants $C_{1}, \ldots, C_{4}$, the eigenfunction describing the $n$-th mode takes its final form

$$
W_{n}(y)=\delta_{n}\left(-\sinh \beta_{n} y+\sin \beta_{n} y+\frac{\sin \beta_{n} l+\sinh \beta_{n} l}{\cos \beta_{n} l+\cosh \beta_{n} l}\left(\cosh \beta_{n} y-\cos \beta_{n} y\right)\right)
$$

where: $\beta_{1} l=1.875, \beta_{2} l=4.694$, and for $n \geqslant 3 \beta_{n} l=(2 n-1) \pi / 2, \delta_{n}$ is the coefficient normalising the $n$-th mode, expressed by the formula

$$
\delta_{n}=\frac{-(-1)^{n}}{\max _{y}\left|W_{n}(y)\right|}
$$


Successive natural frequencies are determined from equation $(3.3)_{2}$

$$
\omega_{n}=\left(\beta_{n} l\right)^{2} \sqrt{\frac{E I}{\rho A l^{4}}}
$$

After determination of the eigenfunction $W_{n}(y)$ and the natural frequency $\omega_{n}$, Eq. $(3.1)_{1}$, describing the beam transverse vibrations is expanded. Energy dissipated is accounted for in the equation assuming a viscoelastic Voigt-Kelvin model describing the properties of the beam material

$$
\sigma\left(y, z_{n l}, t\right)=E \varepsilon\left(y, z_{n l}, t\right)+\eta E \dot{\varepsilon}\left(y, z_{n l}, t\right)
$$

The normal stresses $\sigma$ present in the beam cross-section are dependent on the $y$ coordinate of the cross-section, the distance $z_{n l}$ from the neutral layer, and time $t$. The material internal damping is described by the loss coefficient $\eta$. Thus, the equation of the beam free vibrations with internal damping is as follows

$$
\frac{\partial^{2} w}{\partial t^{2}}+c^{2} \frac{\partial^{4} w}{\partial x^{4}}+\eta c^{2} \frac{\partial^{5} w}{\partial x^{4} \partial t}=0
$$

In this study, forced beam vibrations induced by the movement of the holder along the $z$ axis are studied (Sapiński et al., 2011). A diagram of the beam with kinematic excitation is shown in Fig. 5. The displacement of the holder is described by the variable $u(t)$, and displacement of an arbitrary beam cross-section is described by the variable $w(y, t)$

$$
w(y, t)=z(y, t)+u(t)
$$

where $z(y, t)$ is the displacement related to bending of the beam described by means of the series

$$
z(y, t)=\sum_{n=1}^{\infty} W_{n}(y) q_{n}(t)
$$

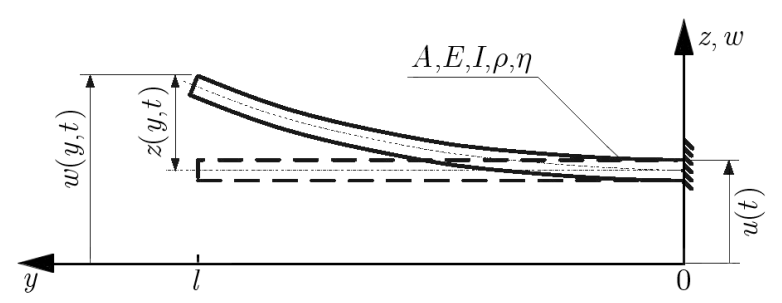

Fig. 5. Scheme of the beam with kinematic excitation

In the above equation, $q_{n}(t)$ is the modal coordinate of the $n$-th mode of vibrations, and $W_{n}(y)$ is the eigenfunction describing the $n$-th mode given by formula (3.6). After inserting equations (3.11) and (3.12) into (3.10), the following is obtained

$$
\sum_{n=1}^{\infty}\left(W_{n} \ddot{q}_{n}+c^{2} W_{n}^{I V} q_{n}+\eta c^{2} W_{n}^{I V} \dot{q}_{n}\right)=-\frac{\partial^{2} u}{\partial t^{2}}
$$

and after accounting for equation $(3.3)_{2}$ and the harmonic excitation expressed in an exponential form $\widetilde{u}(t)=U_{0} \mathrm{e}^{\mathrm{i} \Omega t}$

$$
\sum_{n=1}^{\infty}\left(\ddot{\widetilde{q}}_{n}+\eta c^{2} \beta_{n}^{4} \dot{\widetilde{q}}_{n}+c^{2} \beta_{n}^{4} \widetilde{q}_{n}\right) W_{n}=U_{0} \Omega^{2} \mathrm{e}^{\mathrm{i} \Omega t}
$$


The $(\cdot)$ symbol designates the complex notation with understanding that if the excitation is $u(t)=U_{0} \cos \omega t$, the response is $\operatorname{Re} \widetilde{z}(y, t)$ and if $u(t)=U_{0} \sin \omega t$, the response is $\operatorname{Im} \widetilde{z}(y, t)$. After transformation, taking into account orthogonality of the eigenfunctions, the equation of the $n$-th form forced vibration is obtained

$$
\ddot{\widetilde{q}}_{n}+2 \zeta_{n} \omega_{n}^{2} \dot{\widetilde{q}}_{n}+\omega_{n}^{2} \widetilde{q}_{n}=f_{n} \Omega^{2} \mathrm{e}^{\mathrm{i} \Omega t} \quad f_{n}=\frac{\int_{0}^{l} W_{n} d x}{\int_{0}^{l} W_{n}^{2} d x} U_{0} \quad \zeta_{n}=\frac{\eta \omega_{n}}{2}
$$

The $\zeta_{n}$ quantity is the dimensionless damping coefficient of the $n$-th mode. The solution to equation $(3.15)_{1}$ in the steady state is

$$
\widetilde{q_{n}}(t)=Q_{n}(\mathrm{i} \Omega) \mathrm{e}^{\mathrm{i} \Omega t}
$$

After inserting the solution of $\widetilde{q_{n}}(t)$ to equation $(3.15)_{1}$, the following is obtained

$$
Q_{n}(\mathrm{i} \Omega) X_{n}(\mathrm{i} \Omega)=\Omega^{2} f_{n} \quad X_{n}(\mathrm{i} \Omega)=-\Omega^{2}+2 \zeta_{n} \omega_{n}^{2} \mathrm{i} \Omega+\omega_{n}^{2}
$$

Based on equation $(3.16), \widetilde{q}_{n}(t)$ is determined

$$
\widetilde{q}_{n}(t)=Q_{n}(\mathrm{i} \Omega) \mathrm{e}^{\mathrm{i} \Omega t}=f_{n} G_{n} \mathrm{e}^{\mathrm{i} \Omega t}
$$

where the function $G_{n}(\mathrm{i} \Omega)$ is defined as the following quotient

$$
G_{n}(\mathrm{i} \Omega)=\frac{Q_{n}(\mathrm{i} \Omega)}{f_{n}}=\frac{1}{-1+2 \zeta_{n} \mathrm{i} \frac{\omega_{n}}{\Omega}+\left(\frac{\omega_{n}}{\Omega}\right)^{2}}
$$

After determination of the phase angle $\varphi_{n}$ and the modulus of the function $G_{n}(\mathrm{i} \Omega)$, the modal coordinate $\widetilde{q}_{n}(t)$ is defined as

$$
\widetilde{q}_{n}(t)=f_{n}\left|G_{n}(\mathrm{i} \Omega)\right| \mathrm{e}^{\mathrm{i}\left(\Omega t+\varphi_{n}\right)} \quad \varphi_{n}=\arctan \frac{\operatorname{Im} G_{n}(\mathrm{i} \Omega)}{\operatorname{Re} G_{n}(\mathrm{i} \Omega)}
$$

Ultimately, after inserting equations $(3.20)_{1}$ to (3.12) and determination of the real part from the $\widetilde{z}(y, t)$ function describing the transverse displacement of the beam, the following is obtained

$$
z(y, t)=\sum_{n=1}^{\infty} W_{n}(y) f_{n}\left|G_{n}(\mathrm{i} \Omega)\right| \cos \left(\Omega t+\varphi_{n}\right)
$$

This is an equation describing the beam forced vibrations under harmonic excitation.

Over the course of the beam transverse vibrations, an arbitrary point of the neutral layer is displaced in both the horizontal and vertical directions. Point $P_{r}$ in the neutral layer of the beam and its trajectory $(r=0,1, \ldots, 6)$ are schematically illustrated in Fig. 6 . To determine the trajectory of an arbitrary point $P_{r}$ of the beam, a function describing the displacement of the selected point along the $y$ and $z$ axes must be defined. To determine the vertical position, the formulas derived in the first part of theoretical considerations are used. Through multiplication of normalised eigenfunction (3.6) by the maximum amplitude of transverse vibrations $Z_{r}^{n}$, the following formula is obtained

$$
\widetilde{\widetilde{W}}_{n}(y)=Z_{r}^{n} W_{n}(y)
$$

Successive modes are designated by the index $n=1,2,3$. Within the frequency range of $(9.15,165.71) \mathrm{Hz}$ in which the beam is studied, the appearance and influence of the beam longitudinal vibrations on point trajectories can be eliminated. Consequently, the length of the beam 


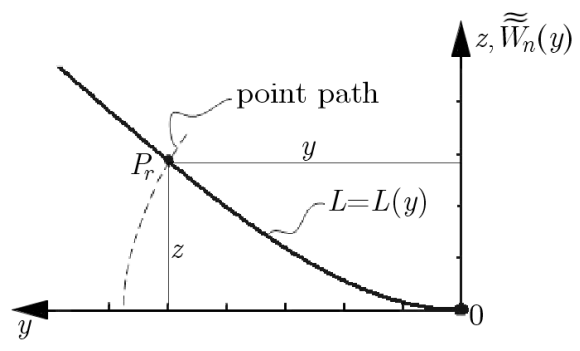

Fig. 6. Trajectory of an arbitrarily selected beam point

is unchanged. In light of the above, the formula for the length of a curve is used to determine the position of point $P_{r}$, which takes the following form in the analysed example

$$
L(y)=\int_{0}^{y} \sqrt{1+\left(\frac{d \widetilde{\widetilde{W}}_{n}(y)}{d y}\right)^{2}} d y
$$

Ultimately, dependencies describing the motion of an arbitrary point of the beam on the plane $y z$ are obtained

$$
z=\widetilde{\widetilde{W}}_{n}(y) \quad L(y)=\int_{0}^{y} \sqrt{1+\left(\frac{d \widetilde{\widetilde{W}}_{n}(y)}{d y}\right)^{2}} d y
$$

\section{Simulation and laboratory studies}

The beam vibrations with kinematic excitation are registered for three frequencies $f$. The displacements $y(t)$ and $z(t)$ are determined for each point $P_{0}-P_{6}$. Examples of the registered displacements in the direction of the $y$ and $z$ axes of point $P_{2}$ for two excitation frequencies are shown in Fig. 7. It is observed that the analysed point also moves along the beam axis in addition to the transverse motion along the $z$ axis. Transverse displacements have a greater value on the negative $z$ axis side. This is related to action of the force of gravity and static deflection of the beam. In reality, a beam in equilibrium is never positioned precisely on the $y$ axis; it only has a certain displacement related to the static deflection. Displacements in the direction of the $y$ axis are also greater on the side of negative values. The greatest amplitudes $y(t)$ are observed when the beam is maximally displaced in the direction of negative values on the $z$ axis, or in accordance with the acting force of gravity (Fig. 7a).

During forced vibrations with a frequency of $f=61.12 \mathrm{~Hz}$, the second form of vibration is dominant in the system. This is made evident by the fact that the first anti-node occurs at point $P_{2}$. For this mode, however, the maximum vibration amplitudes occur at point $P_{6}$ (second anti-node). The greatest displacements in the direction of the $y$ axis are also observed for this point. In relation to the extreme location at point $P_{2}$ (the angle of rotation of the beam cross-section is equal to 0 ), the observed maximum displacement of point $P_{2}$ in the direction of the $y$ axis is equal to about $0.3 \mathrm{~mm}$. These displacements are comparable to those observed for point $P_{4}$, at which a node is present. Based on the displacements $y(t)$ and $z(t)$, the trajectory of motion of points in the plane of the beam vibrations is determined. Examples of the trajectories of point $P_{2}$ are presented in Fig. 8 .

In the next stage of the study, the goal is to verify the modes of vibrations obtained from theoretical considerations against the modes of vibrations obtained from the experiment. The question is whether the amplitudes of transverse displacements $Z_{1}-Z_{6}$ of the corresponding points $P_{1}-P_{6}$ would coincide with the amplitudes determined on the basis of theoretical considerations. 

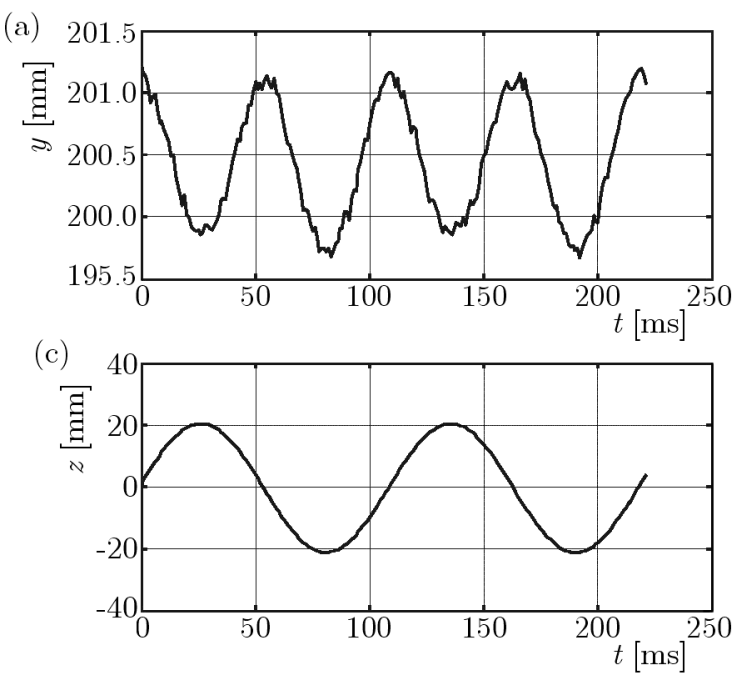

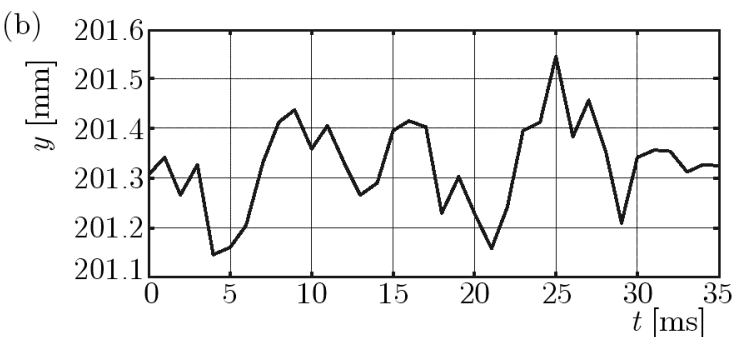

(d)

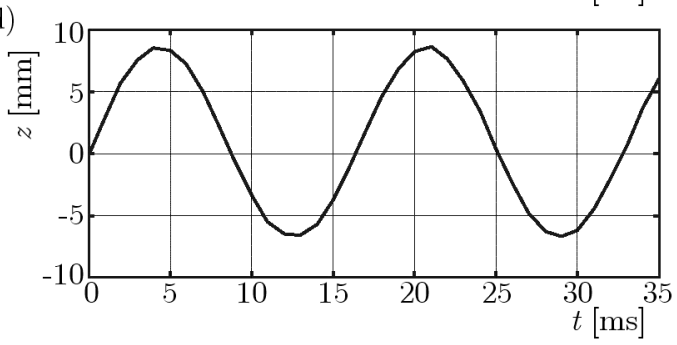

Fig. 7. Time histories $y(t)$ and $z(t)$ of point $P_{2}$ : (a) $f=9.15 \mathrm{~Hz}$, (b) $f=61.12 \mathrm{~Hz}$
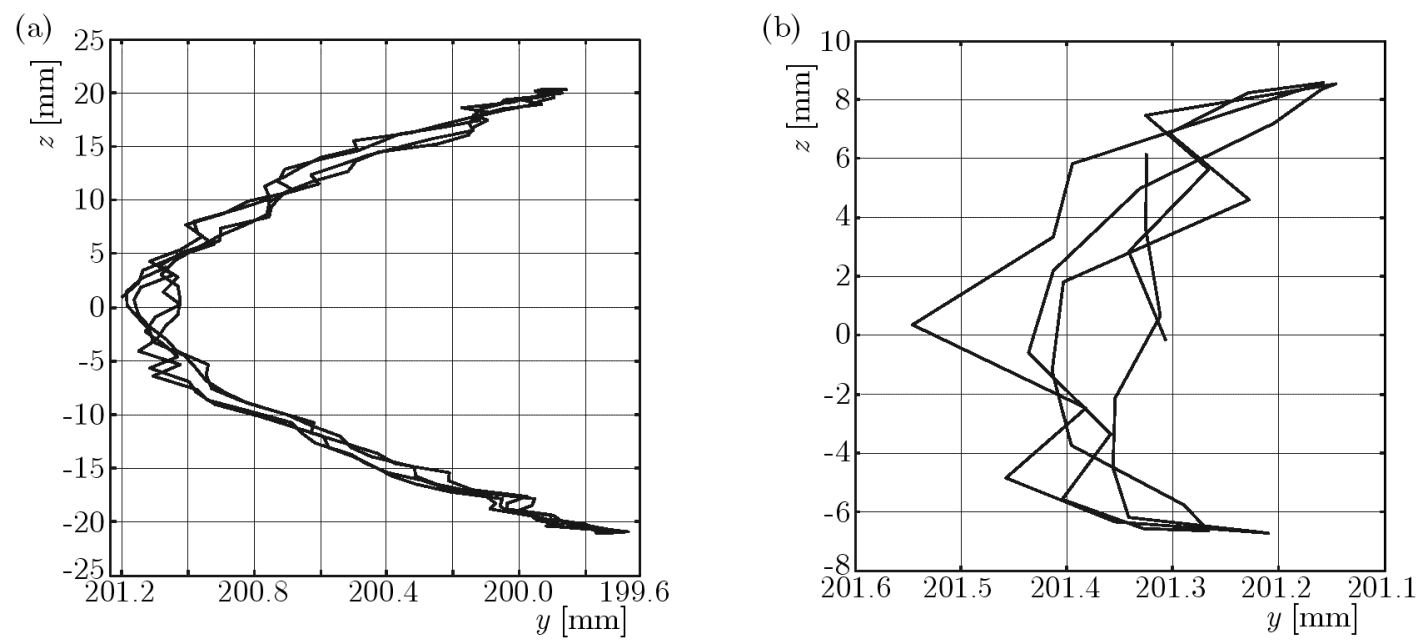

Fig. 8. Trajectory of point $P_{2}$ in the $y z$ plane: (a) $f=9.15 \mathrm{~Hz}$, (b) $f=61.12 \mathrm{~Hz}$

Amplitudes have been measured relative to the accepted coordinate system yz (Figs. 2 and 3). For this purpose, functions describing the modes for a homogeneous cantilever beam are used (3.6). The maximum amplitude $Z_{6}^{n}$ of the beam transverse vibrations is read for every recorded form. In this way, a formula enabling the determination of the amplitude of an arbitrary point $P$ with coordinates $P=(y, 0)$ for $y \in\langle 0,400\rangle \mathrm{mm}$ is obtained

$$
\widehat{W}_{n}(y)=Z_{6}^{n} \widehat{\delta}_{n} W_{n}(y) \quad n=1,2,3
$$

The succesively read amplitudes $Z_{6}^{1}, Z_{6}^{2}$ and $Z_{6}^{3}$ are equal to $57.5,10.1$ and $4.6 \mathrm{~mm}$. In view of the fact that point $P_{6}$ is situated at a distance of $396.4 \mathrm{~mm}$ from the beginning of the beam, a modified formula for normalising coefficient $\widehat{\delta}_{n}$ is obtained

$$
\widehat{\delta}_{n}=\frac{-(-1)^{n}}{\max _{y \in\langle 0,396.4\rangle}\left|W_{n}(y)\right|}
$$

The results obtained from the recording of seven points $P_{0}-P_{6}$ by the vision system are compared to the results obtained using formula (3.6). The vibration modes of beams are presented in Fig. 9: first, second, and third, determined on the basis of formula (3.22) and experimental studies. The broken line is used to mark natural vibration modes determined using the model, 
and circles are used to mark deviations for the successive measuring points $P_{0}-P_{6}$. Figure 9 shows, for forced beam vibrations observed by means of the vision system, that the modes of the beam are consistent with the presented models. Nodes and antinodes are present at the measuring points $P_{0}-P_{6}$, as established at the outset. The presented results confirm the correctness of the conducted experiments. In addition, they show the enormous possibilities and advantages of using the presented vision system to record and analyse vibrations of continuous systems. This method enables simultaneous observation of characteristic points of the tested object and recording the parameters of motion of these points on a plane.
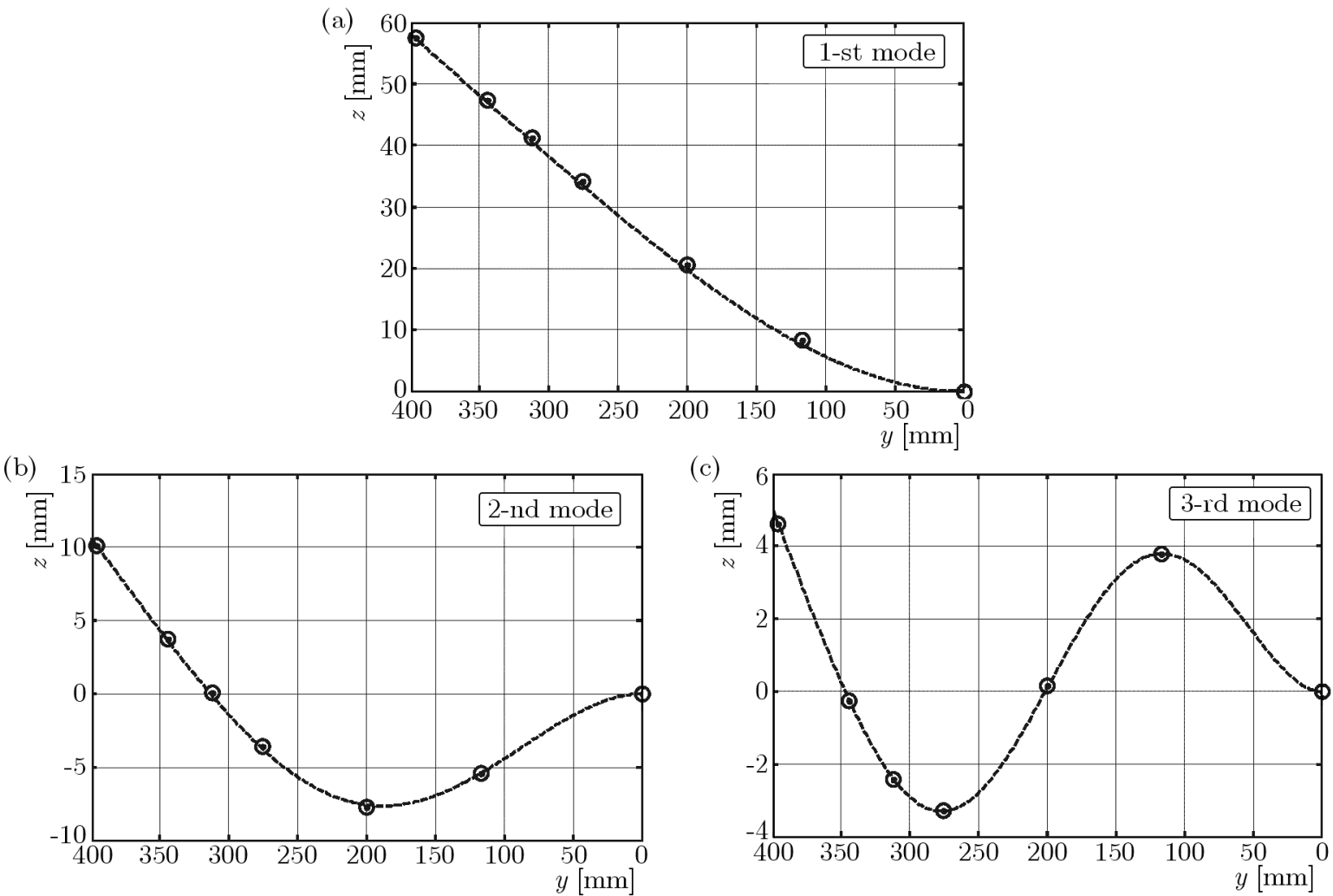

Fig. 9. First three modes of beam vibrations
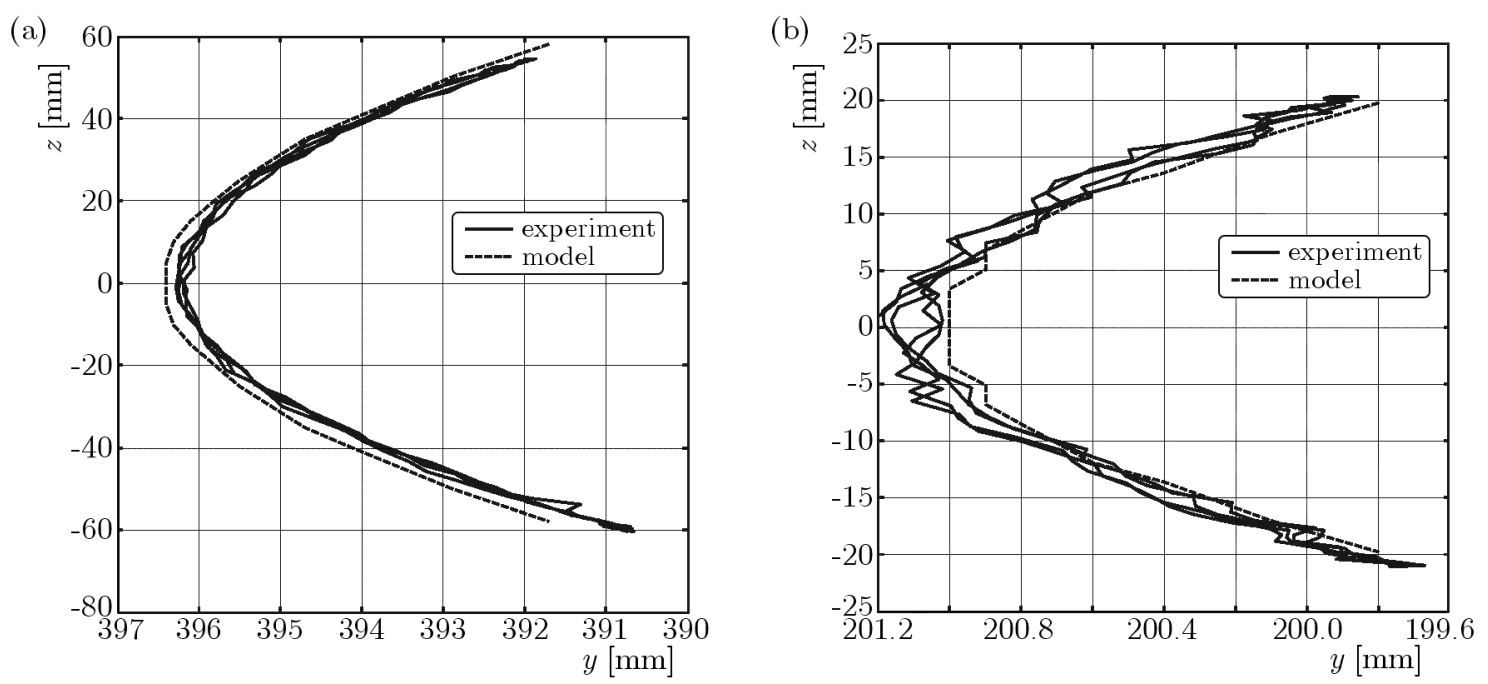

Fig. 10. Trajectory of point: (a) $P_{6}$ and (b) $P_{2}$ in the $y z$ plane for the first mode $(f=9.15 \mathrm{~Hz})$ 
During the final stage of studies, the trajectories of six points of the beam $\left(P_{1}-P_{6}\right)$ are determined for every excitation frequency by numerically solving the system of equations (3.24). Examples of trajectories obtained from the system of equations (3.24) and from the experiment are shown in Fig. 10. The presented trajectories show the concurrence of results obtained from the theoretical considerations with those obtained from the experiment. The proposed model correctly analyses the possible motion of arbitrary points of the beam over duration of its forced vibrations. Discrepancies between the model and experiment do not exceed $1 \%$. It is also observed that, for amplitudes of the transverse vibrations of point $P_{6}$ less than $20 \mathrm{~mm}$ (Fig. 10a) and vibrations of point $P_{2}$ less than $6 \mathrm{~mm}$ (Fig. 10b), the displacements along the $y$ axis are very small. The beam points $\left(P_{0}-P_{6}\right)$ for small amplitudes $\left(Z_{1}-Z_{6}\right)$ do not change their position in the $y$ axis.

\section{Conclusions}

Analysis of vibrations of a homogeneous cantilever beam subjected to kinematic excitation with a moving holder has been conducted with the application of a vision method. The configuration of the vision system and the test stand for study of forced beam vibrations have been presented. Seven measuring points situated on the beam have been selected for analysis. The algorithm for processing the recorded image of beam vibrations and determining the displacements of the defined points has been discussed. A model describing forced beam vibrations, taking internal material damping into account, has been presented. Formulas defining the trajectory of arbitrary points of the beam have been derived. Images of beam vibrations have been recorded with observation of the first three modes of vibrations. The displacements of the defined measuring points of the beam have been determined on the basis of measurements. Very strong concurrence of the experimental results with the results obtained from the model has been obtained. The application of the vision method enables contactless measurement and makes it possible to observe displacements of many measuring points of the beam in the studied plane of vibrations.

Acknowledgments

This work has been supported by AGH University of Science and Technology under statutory research program No. 11.11.130.958.

\section{References}

1. Chen F., Luo W.D., Dale M., Petniunas A., Harwood P., Brown G.M., 2003, High-speed ESPI and related techniques: overview and its application in the automotive industry, Optics and Lasers in Engineering, 40, 5/6, 459-485

2. Freymann R., Honsberg W., Winter F., Steinbichler H., 1996, Holographic modal analysis, [In:] Laser in Research and Engineering, Springer-Verlag, Berlin, 530-542

3. Gawlik J., Ryniewicz A., Sioma A., 2004, The strategies and methods of measurement in multifunctional quality inspection, 8th International Symposium on Measurement and Quality Control in Production, Erlangen, Measure and Quality Control in Production Book Series: VDI Berichte, 1860, 649-662

4. Konut P., Kurowski P., 2005, The integration of vision based measurement system and modal analysis for detection and localization of damage, 21st International Conference on CAD/CAM, Robotics and Factories of the Future CARS\&FOF 2005, Krakow, 391-398

5. Kohut P., Kurowski P., 2009, Application of modal analysis supported by 3D vision-based measurements, Journal of Theoretical and Applied Mechanics, 47, 4, 855-870 
6. Kowal J., Sioma A., 2009, Active vision system for 3D product inspection: Learn how to construct three-dimensional vision applications by reviewing the measurements procedures, Control Engineering, 56, 11, 46-48

7. Ma Y., Soatto S., Kostecka J., Sastry S., 2004, An Invitation to 3D Vision, Springer-Verlag, New York

8. Meirovitch L., 1997, Principles and Techniques of Vibrations, Prentice Hall, Englewood Cliffs, New York

9. Meirovitch L., 2001, Fundamentals of Vibrations, McGraw-Hill, New York

10. Moreno D., Barrientos B., Pérez-López C., Mendoza Santoyo F., 2005, Modal vibration analysis of a metal plate by using a laser vibrometer and the POD method, Journal of Optics a: Pure and Applied Optics, 7S356-S363

11. Peeters B., Peeters K., Van der Auweraer H., Olbrechts T., Demeester F., Wens L., 2004, Experimental modal analysis using camera displacement measurements: a feasibility study, Proceedings of the SPIE the International Society for Optical Engineering, 5503, 298-309

12. Romaszko M., PakuŁa S., Sapiński B., Snamina J., 2011, Vibration parameters of a sandwich beams with two types of MR fluid, Mechanics and Control, 30, 3, 151-156

13. Sapiński B., Snamina J., Romaszko M., 2010, Identification of model parameters of a sandwich beam incorporating magnetorheological fluid, Vibrations in Physical Systems, 24, 349-354

14. Sapiński B., Snamina J., Romaszko M., 2011, Wyniki badań doświadczalnych drgań wymuszonych belki z cieczą magnetoreologiczną, Czasopismo Techniczne, 108, 2, 215-222

15. Schmidt T., Tyson J., Galanulis K., 2003, Full-field dynamic displacement and strain measurement using advanced 3D image correlation photogrammetry, Experimental Techniques, 27, 3, 41-44

16. Sioma A., 2013, The rope wear analysis with the use of $3 \mathrm{D}$ vision system, Control Engineering, 60, $5,48-49$

17. Snamina J., Sapiński B., Romaszko M., 2012a, Vibration parameter analysis of a sandwich cantilever beams with multiple MR fluid segments, Engineering Modeling, 43, 247-254

18. Snamina J., Sapiński B., WszoŁek W., Romaszko M., 2012b, Investigation on vibrations of a cantilever beam with magnetorheological fluid by using the acoustic signal, Acta Physica Polonica A, 121, 1-A

19. Sriram P., Hanagud S., Craig J.I., 1992, Mode shape measurement using a scanning laser Doppler vibrometer, The International Journal of Analytical and Experimental Modal Analysis, 7, $3,169-178$

20. Trucco E., Verri A., 1998, Introductory Techniques for 3D Computer, Vision, Prentice-Hall

21. Van der Auweraer H., Steinbichler H., Haberstok C., Freymann R., Storer D., 2002, Integration of pulsed-laser ESPI with spatial domain modal analysis, Shock and Vibration, 9, 1/2, $29-42$ 